\section{$\underset{\substack{\text { hommes } \\ \text { \& migrations }}}{ }$}

\section{Hommes \& migrations}

Revue française de référence sur les dynamiques

migratoires

1307 | 2014

L'Afrique qualifiée dans la mondialisation

\title{
Les Bamakois diplômés de Paris
}

Ethnographie d'une petite bourgeoisie en voie de déclassement

\section{David Mahut}

\section{(2) OpenEdition}

1 Journals

\section{Édition électronique}

URL : http://journals.openedition.org/hommesmigrations/2872

DOI : $10.4000 /$ hommesmigrations.2872

ISSN : 2262-3353

Éditeur

Musée national de l'histoire de l'immigration

\section{Édition imprimée}

Date de publication : 1 juillet 2014

Pagination : 19-27

ISBN : 978-2-919040-28-5

ISSN : $1142-852 X$

\section{Référence électronique}

David Mahut, «Les Bamakois diplômés de Paris », Hommes \& migrations [En ligne], 1307 | 2014, mis en ligne le 01 juillet 2017, consulté le 02 mai 2019. URL : http://journals.openedition.org/

hommesmigrations/2872 ; DOI : 10.4000/hommesmigrations.2872 


\title{
LES BAMAKOIS DIPLÔMÉS DE PARIS ETHNOGRAPHIE D'UNE PETITE BOURGEOISIE EN VOIE DE DÉCLASSEMENT
}

par DAVID MAHUT, sociologue, chercheur associé à l'UMR CEPED, université de Paris-Descartes.

\begin{abstract}
En France, ils sont chauffeurs-livreurs, commis de cuisine, veilleurs de nuit, infirmiers ou préparateurs en pharmacie. Pourtant, leurs diplômes obtenus dès la fin des années 1990 dans un Mali en crise les préparaient à d'autres emplois. Certains diplômés maliens subissent un déclassement socioprofessionnel aux racines profondes, qui pointe les impasses respectives des sociétés française et malienne en termes de mobilité sociale. Devant la non-reconnaissance de leurs diplômes dans l'Hexagone, face à la nécessité de se faire une situation pour apporter leur contribution financière à leur famille, ils acceptent non sans mal de devenir ouvriers ou employés.
\end{abstract}

Cette étude du déclassement dans la migration repose sur une enquête ethnographique menée entre 2007 et 2012 auprès d'une quarantaine de migrants bamakois et diplômés résidant à Paris ${ }^{1}$. Ils forment un groupe d'interconnaissance et présentent la particularité d'être originaires de la petite bourgeoisie bamakoise. Issus d'un milieu social aisé, ils sont aussi porteurs d'un capital scolaire relativement important puisque le niveau de leurs diplômes obtenus au Mali varie du baccalauréat au doctorat, ce qui implique une maîtrise parfaite de la langue française ${ }^{2}$. Malgré ce capital prémigratoire spécifique ${ }^{3}$, la majorité des enquêtés restent en bas de la hiérarchie des métiers, ce qui est en inadéquation avec leur niveau de qualification. Mais ce déclassement socioprofessionnel n'est pas le résultat de leur immigration en France. Il a débuté dans le Mali des années 1990, alors que le pays traversait une crise qui gelait leurs possibilités d'ascension professionnelle et familiale.

\section{La petite bourgeoisie bamakoise}

Afin de comprendre la spécificité du milieu social d'origine des enquêtés, il faut garder à l'esprit que la petite bourgeoisie bamakoise fonde ses méca- 
nismes de reproduction sociale sur deux composantes : la dette intergénérationnelle et le diplôme. Pour mes interlocuteurs, la dette entre les générations $s^{4}$ est au cœur du système de protection microsocial malien. Il repose sur une forte hiérarchisation du groupe de parenté selon le genre et l'âge de ses membres. Au sommet de la pyramide familiale se trouve le chef de famille, le "patriarche dominateur", pour reprendre l'expression de Seydou Badian Kouyaté $^{5}$. D'un point de vue normatif, ce statut est réservé aux hommes.

Selon le principe de séniorité ouverte ${ }^{6}$, la hiérarchie familiale s'ordonne de manière à ce que chaque homme puisse franchir les étapes qui conduisent au statut d'ainé. Les ascendants pourvoient à l'ensemble des nécessités vitales et des besoins socioéconomiques de leurs enfants. Ces derniers nourris, soignés, hébergés, Dès le début éduqués - s'inscrivent alors des années 1980, le Mali entre du côté des débiteurs.

dans une crise qui La "dette" ainsi contractée bouleverse les équivalences éducatives du passé. donne aux créanciers, les aînés, une assurance sur l'avenir. Aussitôt que les descendants sont en mesure de rendre la pareille, ils sont tenus de manifester leur reconnaissance à ceux dont ils ont reçu par le passé (sous la forme de prestations de travail ou monétaires). C'est précisément ce qui, pour les enquêtés, caractérise le passage à lâge adulte : le moment où, en devenant un membre actif du collectif familial, on apporte "sa contribution à la famille" (Issa, 53 ans, médecin à Bamako). Enfin, un nouvel échelon de la hiérarchie familiale est franchi lorsqu'on se marie et qu'on devient père, "parce que c'est à tes enfants de te donner quelque chose, de donner quelque chose au papa" (Ibrahim, 38 ans, docteur en pharmacie).

D’abord uniquement débiteurs, les adultes deviennent à leur tour créanciers en endettant leurs obli- gés, en amorçant un nouveau cycle d'avances-restitutions ${ }^{7}$. Ainsi résumée, la logique de la dette est censée garantir la continuité des générations et permettre aux mécanismes de reproduction domestique de fonctionner. Quant aux modalités du contrat générationnel, elles s'établissent selon le milieu social considéré. C'est ici que le diplôme tient un rôle considérable.

$\mathrm{Au}$ lendemain de l'indépendance du Mali, les pères des enquêtés ont mis à profit leur capital scolaire rare dans les années 1960-1970 - pour accéder aux professions libérales (avocat, médecin, pharmacien, etc.). Cette forme d'intégration professionnelle a donné la possibilité à ces pères d'inscrire leur famille parmi les fractions privilégiées et très minoritaires de la population bamakoise : niveau des revenus (au minimum cinq fois supérieur au salaire minimum malien ${ }^{8}$ ), métiers intellectuels (par opposition aux métiers manuels), consommation de luxe (dans l'habillement, les loisirs, les transports), patrimoine immobilier, scolarisation longue de leurs enfants ${ }^{9}$. Mais surtout, leurs revenus d'activité ont permis de soutenir le système de protection sociale basé sur la dette intergénérationnelle. Ainsi, la promotion par le diplôme leur a garanti une ascension à la fois professionnelle et familiale. Mes interlocuteurs n'avaient plus quà suivre le modèle de réussite de leurs pères.

\section{Les raisons du départ}

Alors que l'ascenseur social - par le biais de l'école a fonctionné durant vingt ans, la ville de Bamako a connu dans le même temps de profondes métamorphoses : explosion démographique, expansion urbaine, demande accrue de scolarisation. Dès le début des années 1980, le Mali entre dans une crise qui bouleverse les équivalences éducatives du passé.

4. Alain Marie, L’Afrique des individus, Paris, Karthala, 1997. 5. Conférence de Seydou Badian Kouyaté (ancien Premier ministre du Mali et écrivain) dans l'école privée Moribougou, Journal de terrain, Bamako, le 6 décembre 2007. 6. Georges Balandier, Anthropo-logiques, Paris, Le Livre de poche, 1985, p. 132. 7. Claude Meillassoux, Femmes, greniers et capitaux, Paris, L'Harmattan, 1992. 8. Les revenus liés aux professions libérales s'élèvent en moyenne à 200000 francs CFA par mois (300 euros) selon le ministère de l'Emploi et de la Formation professionnelle (Bilan de l'emploi 2007, Bamako, 2007). Le salaire minimum au Mali varie entre 21936 francs CFA (33 euros) et 35 049 francs CFA (53 euros) par mois (article D.86-10 du décret 96-178 du 13 juin 1996). 9. La proportion d'hommes ayant obtenu le baccalauréat ne dépassait pas les 3\% en 2006, ministère de l'Économie, de l'Industrie et du Commerce, Enquête démographique et de santé du Mali, 2006 , Bamako, EDSM-IV, 2007, p.18. 


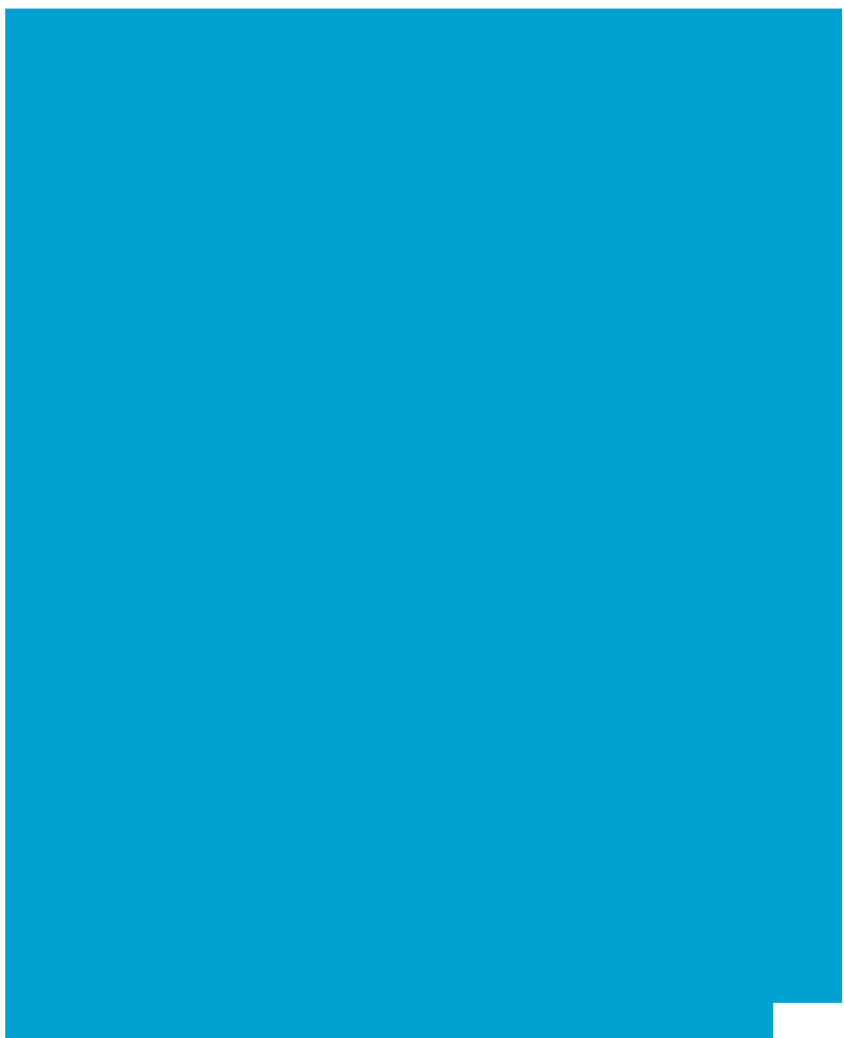

commencer par l'impossibilité de contribuer dignement à l'économie domestique, c'est-àdire à la hauteur de la condition sociale de leur famille: "J'ai été pompiste plusieurs mois. Je disais rien à mes parents. Attends, mon père est médecin, il a bonne réputation. Et puis c'est pas avec l'argent que je gagnais que je pouvais prendre le relais" (Gaoussou, master 1 en économie). La participation financière dans le groupe de parenté - alors inexistante ou insuffisante - constituait pourtant le point de passage obligé pour gagner le statut d'adulte. Âgés entre 20 et 30 ans, mes interlocuteurs ont vu leurs chances de mobilité familiale se volatiliser.

Le temps social des enquêtés a été différent de celui de leurs parents, ne serait-ce que parce que le diplôme n'avait plus le privilège de la rareté. C'est le dysfonctionnement des mécanismes de reproduction domestique, fondés sur la dette et le diplôme, sur la hiérarchie familiale et professionnelle, qui a empêché l'héritage des positions sociales.

Beaucoup d'étudiants rencontrés reconnaissent que sur la forme, des changements ont lieu, mais sur le fond, des problèmes demeurent. Parmi eux, sont souvent cités les problèmes d'accès au restaurant universitaire et le manque de transports en commun. Abidjan, mai 2014 (c Camille Millerand

Lorsque les enquêtés terminent leurs études, le marché du travail bamakois est à la fois saturé (il y a beaucoup plus de candidats diplômés que de places disponibles) et bridé par les politiques d'ajustements structurels ${ }^{10}$. Alternant entre des périodes de chômage et des petits boulots intermittents, mes interlocuteurs ne parviennent pas à s'insérer dans les domaines correspondant à leur formation (Fonction publique, enseignement, recherche, santé, etc.).

Cette situation face à l'emploi n'a pas seulement été synonyme d'un déclassement professionnel. Elle s'est répercutée sur l'ensemble de leur vie sociale, à
Alors seulement, mes interlocuteurs ont songé à l'alternative migratoire ${ }^{11}$.

\section{La reproduction sociale de l'immigration par le bas}

Les Bamakois diplômés rencontrés à Paris ont migré entre 1999 et 2007. Presque tous ont été accueillis par des membres de leur famille : un oncle, un frère, un parent. Ce mode familial d'entrée dans la société française a été décisif pour la suite de leur itinéraire professionnel. Les situations d'accueil rapportées font mention d'une prise en charge partielle du réseau migrant : irrégularité des temps d'hébergement, soutien matériel et financier ponctuel selon les ressources disponibles. En l'espace de quelques mois, les enquêtés sont placés sur les marges du groupe d'accueil, à la limite 
de la zone d'assistance qu'il était censé garantir. Les conséquences sont immédiates : difficultés économiques, périodes de solitude, affaiblissement des relations sociales stables, durables et quotidiennes. En se remémorant les premiers temps de sa vie à Paris, Ousmane déclare : "Être immigré... j’avais l'impression que cétait comme une longue maladie à gérer" (32 ans, bachelier). Cette fragilisation rapide des conditions d'immigration contraint les enquêtés à travailler au plus vite pour atteindre une autonomie financière et résidentielle. Là encore, le groupe d'accueil occupe une place importante, puisque tous les enquêtés sont passés par lui pour trouver leur premier emploi. Cette aide à l'embauche est largement déterminée par la position professionnelle des "accueillants". Or ceux-ci sont majoritairement ouvriers ou employés. Pris par la nécessité économique, mes interlocuteurs sont nombreux à avoir renoncé à faire un usage professionnel de leurs titres universitaires et à sêtre dirigés vers les emplois du bas de l'échelle et du marché secondaire du travail ( $C D D$ et intérim).

\section{Un marché du travail pour immigrés}

Le mode familial d'entrée dans la vie active a imposé aux enquêtés un point départ dans leur itinéraire professionnel situé en bas de la hiérarchie des métiers ; comme si l'inscription dans le réseau migrant, bien que partielle, suffisait à enclencher un mécanisme de transmission des positions sociales dans l'immigration. Cela dit, leur situation professionnelle à Paris ne peut pas être pleinement comprise sans évoquer succinctement les contraintes juridiques et sociales liées à la condition immigrée : système des titres de séjour, invalidation du diplôme étranger, emplois réservés aux Français, division ethnique du travail, etc. Sans entrer dans les détails du processus historique de la formation d'un "marché du travail pour immigrés"12", il est indé- niable que les contraintes qui lui sont attachées, et surtout leur combinaison, sont essentielles pour expliquer la surreprésentation des enquêtés dans des emplois souvent précaires, où la qualification requise est presque toujours inférieure au niveau de leurs diplômes ${ }^{13}$. Aujourd'hui, mes interlocuteurs exercent des métiers d'ouvrier et d'employé (chauffeur-livreur, commis de cuisine, veilleur de nuit, etc.) ou se rapprochent des catégories professionnelles intermédiaires (infirmier, préparateur en pharmacie, professeur dans une association). En recoupant les salaires nets et mensuels de ces activités avec les différentes strates du salariat en France, ils appartiendraient à la classe populaire (avec un salaire inférieur ou égal à $1143 €$ ) et à la classe moyenne inférieure (avec un salaire compris entre 1143 et $1524 €)^{14}$, dont les conditions de vie ne sont plus celles de la petite bourgeoisie bamakoise.

\section{Peut-on parler de déclassement?}

L'utilisation de la notion de déclassement présente au moins deux difficultés. La première est qu'elle renvoie à différents niveaux de réalité. Généralement, le déclassement désigne une mobilité sociale vers le bas, c'est-à-dire le mouvement qui consiste à descendre un ou plusieurs échelons de la hiérarchie sociale. Il conduit l'individu à s'installer durablement dans une condition socio-économique inférieure à celle qu'il a connue à un moment passé de son existence. Le déclassement générationnel (conditions de vie en-deçà de celles de ses ascendants) entre dans cette définition. Mais le déclassement peut aussi résulter d'un décalage entre la position sociale de l'individu et celle à laquelle il pourrait prétendre étant donné ses dispositions sociales. Le déclassement professionnel (compris au sens d'une inadéquation entre le diplôme et l'emploi) en est un exemple. Ici, le déclassement ne renvoie plus à une mobilité descendante, mais à 
l'impossibilité, pour l'individu, de poursuivre la trajectoire la plus probable compte tenu de son capital scolaire. Bref, le déclassement recouvre plusieurs dimensions auxquelles il faut prêter attention.

La seconde difficulté peut se résumer à une question : comment parler de déclassement lorsque le changement de position sociale s'effectue dans le cadre de la migration? Parler de déclassement implique de considérer à la fois la position que l'individu occupait dans la société d'émigration (le Mali) et celle qu'il occupe dans la société d'immigration (la France). Or cette considération revient à interroger deux modes de hiérarchisation sociale qui ne peuvent pas être confondus.

Tout en réfléchissant aux problèmes que soulève la notion de déclassement appliquée à une population migrante, mais aussi pour tenter de les surmonter, le parti pris est de se pencher sur ce que les enquêtés ont à dire de leur situation d'immigration, en particulier de leur travail. En d'autres termes, pour que le déclassement ait un sens, il faut d'abord que mes interlocuteurs l'aient perçu.

\section{Des rapports différenciés au travail}

Pour explorer - ne serait-ce que partiellement - la relation des enquêtés avec leur travail, je vais comparer les points de vue de Yaya et d'Amadou, qui ont exercé le même métier dans des conditions similaires : celui de chauffeur-livreur en intérim.

Titulaire du baccalauréat, Yaya nourrit une ambition à l'égard de son parcours migratoire: "être indépendant", c'est-à-dire "avoir un salaire, une voiture et un appartement". N'ayant d'autre volonté que d'exercer une activité rémunérée, "peu importe dans quoi", il accepte une mission comme chauffeur-livreur dans une blanchisserie hôtelière parisienne. "Dans latelier, il n'y a que des immigrés. Ils font le nettoyage du linge, le repassage. La plupart ne savent ni lire ni écrire. Là, j’ai compris qu'avec un petit diplôme comme le bac, j'avais plus de chances d'être chauffeur que les autres qui n'avaient pas mon niveau." Selon Yaya, sa maitrise de la langue française lui a été essentielle pour obtenir le poste, ne serait-ce que pour se différencier des migrants qui ne possèdent pas cette compétence. En effet, lire et écrire le français était un critère d'embauche fixé par l'employeur : pouvoir remplir un constat en cas d'accident de la route, lire un plan de Paris, prendre des notes sur les itinéraires de livraison et leurs horaires,
Parler de déclassement implique de considérer à la fois la position que l'individu occupait dans la société d'émigration (le Mali) et celle qu'il occupe dans la société d'immigration (la France). etc. Ainsi, l'entrée de Yaya dans l'entreprise, et plus largement dans la vie active en France, a été favorisée par son capital scolaire acquis avant son émigration.

En outre, la place de chauffeur-livreur autoriserait dans le travail une autonomie plus grande que celle des ouvriers de l'atelier : "Quand je conduis mon camion, il n'y a personne pour me dire quoi que ce soit. Je suis libre de mettre la radio, de téléphoner, de fumer. Au final, je ne vois jamais les patrons. Ça, c'est une grande différence avec ceux qui sont dans l'atelier. En fait, je gère comme je veux. Le tout, c'est de livrer dans les temps. (...) Alors oui, je peux dire que j'aime ce boulot parce que, déjà, j’aime conduire." La faible fréquence des contacts avec ses supérieurs, le degré de liberté dans la gestion de son activité, le plaisir qu'il éprouve à conduire..., plusieurs éléments du métier sont une source de satisfaction pour mon interlocuteur, comme si le temps passé dans "son" camion était un temps à soi, comme s'il y avait du hors-travail dans le travail.

\section{Résister à la violence symbolique}

Pourtant, cette satisfaction qu'éprouve Yaya à être chauffeur-livreur contraste fortement avec la frustration ressentie par Amadou lorsqu'il exerçait ce métier: "Je ne pouvais pas rester chauffeur-livreur. 
Je n'y voyais aucun intérêt! Mais il fallait bien payer le loyer. Alors, faute de mieux... Vous voyez le topo, un petit Noir livreur, catégorisé, typé... Quelquefois, avec ce que les gens ont comme image du Noir, ils se montrent supérieurs. Mais il y a toujours une façon de rabaisser le caquet d'une personne sans utiliser les mêmes armes que lui. Un jour, il y a une dame qui vient: 'Monsieur, vos pompes sont sales.' Je ne vais pas lui dire: 'Madame, cette information est gratuite.' Elle va comprendre, elle va continuer à discuter. Je lui dis : 'Madame, je suis désolé mais votre syllogisme manque de prémisses.' Elle n'a pas compris, elle s'est tue" (40 ans, master 2 en biologie cellulaire). Cet enquêté n'envisage son activité professionnelle que comme une solution d'appoint. Il la perçoit également comme un support de dévalorisation symbolique, notamment parce qu'elle serait propice à l'activation des stéréotypes liés à la couleur de la peau. Lors de ses livraisons, Amadou n'hésite pas à user de ses ressources linguistiques pour déjouer les éventuelles assignations identitaires arbitraires et attitudes stigmatisantes. Une fois de plus, le capital scolaire joue un rôle déterminant : il per-

Considérer le rapport entre le capital prémigratoire, les aspirations et la position sociale des migrants dans la société d'installation est un préalable nécessaire pour pouvoir parler de déclassement

et tenter de cerner ses indicateurs. met à Amadou de résister aux pressions de la "violence symbolique ${ }^{15}$ ".

C'est aussi son capital scolaire qui le conduit à déconsidérer son travail. Sans lien avec sa formation universitaire, ce statut professionnel porte atteinte à son identité de diplômé. Il faudra qu'Amadou se reconvertisse dans l'enseignement pour que se réduise l'écart entre ses aspirations initiales (celles d'être chercheur) et sa position dans l'espace professionnel parisien ${ }^{16}$.

Les cas de Yaya (bachelier) et d'Amadou (bac +5) montrent qu'il n'y a pas "de relation mécanique entre
Espace de révision occupé par des étudiants en économie, université Félix-Houphouët-Boigny (Abidjan), mai 2014. (c) Camille Millerand

les conditions de travail et le degré de satisfaction ou d'insatisfaction ressentie (...). Un travail pourra satisfaire celui pour qui il représente ce qu'il pouvait espérer de mieux. Il mécontentera celui qui le vivra comme un déclassement ${ }^{17 "}$. Considérer le rapport entre le capital prémigratoire, les aspirations et la position sociale des migrants dans la société d'installation est donc un préalable nécessaire pour pouvoir parler de déclassement et tenter de cerner ses indicateurs.

\section{Le diplôme : un double coup d'arrêt}

"Si tu as eu un diplôme en Afrique et que tu es venu en France, ce diplôme-là, il faut le laisser chez toi. C'est vrai, parce que dans le pays où tu es venu, il y en a des 
diplômés! Attends, eux-mêmes ils galèrent, les diplômés français ! À ce moment-là, il faut travailler avec tes mains, pas avec ta tête" (Beidi, 35 ans, BTS en comptabilité).

Manutentionnaire depuis trois ans, Beidi effectue un travail réflexif pour accepter ce qui lui est arrivé et s'habituer à faire avec une situation de déclassement professionnel qu'il pense définitive. Mais cet enquêté introduit un élément important pour expliquer sa situation de travail en France : l'inflation des diplômes. "Durant près de vingt-cinq ans [entre 1950 et 1975], l'ascenseur social a fonctionné sans faiblir pour ceux qui obtenaient des diplômes. Aujourd'hui encore, les jeunes diplômés s'insèrent mieux dans l'emploi que ceux qui n'ont pas de qualifications scolaires. Mais cette loi générale présente de nombreuses failles: les emplois qualifiés ayant crû beaucoup moins rapidement que les diplômes, de plus en plus de jeunes scolairement qualifiés n'accèdent pas aux emplois auxquels ils pensaient pouvoir prétendre ${ }^{18 . "}$

Dans un contexte où les diplômés se multiplient, augmentant la compétition pour les emplois qualifiés, on comprend mieux pourquoi certains interlocuteurs consentent - comme d'autres diplômés en France ${ }^{19}$ - à exercer un travail déclassé : "Mieux vaux ça que d'être au chômage !" (Demba, 26 ans, licence en télécommunications). Cela ne va pas sans rappeler une situation déjà vécue par les enquêtés à Bamako: "Tu viens en France pour fuir une situation et tu te retrouves dans le même merde" (Issa, 27 ans, master en gestion). Pour paraphraser une métaphore utilisée par mes interlocuteurs, le diplôme ne nourrissait pas à Bamako, il ne nourrit pas non plus à Paris. Et c'est la promotion sociale par le diplôme qui subit un second coup d'arrêt.

\section{Les mirages de la carrière}

Plusieurs enquêtés sont confinés dans les emplois du marché du travail secondaire. Travailleurs déclassés, ils sont aussi des travailleurs précaires.
Du point de vue du rapport au travail, cette conjugaison de variables n'est pas sans conséquences. Demba cumule les emplois intérimaires depuis son arrivée en France en 2002. Après avoir raconté les détails d'une dizaine d'expériences professionnelles, il conclut: "Je sais tout faire : électricité, maçonnerie, nettoyage, tout! (...) Non, je ne suis pas satisfait parce que, avec l'intérim, tu ne sais jamais ce que tu feras le lendemain, tu ne sais jamais avec qui tu travailleras... Tu ne peux pas organiser ta vie en fait [silence]. J'ai appris à accepter que, du jour au lendemain, tout peut s'arrêter" (26 ans, licence en télécommunications).

Cette déclaration donne un "Faire carrière", voilà une aperçu d'une vision du travail construite dans l'incertitude et la discontinuité. Elle reflète également une socialisation professionnelle hétérogène (pluralité des lieux de travail, des expression courante qui perd de sa signification quand on n'accède pas à la stabilité de l'emploi et quand on multiplie les expériences de travail sans rapport les unes avec les autres. tâches effectuées, des personnes rencontrées) qui laisse peu de place à la construction d'une identité de travail sur le long terme dans un seul et même métier. Elle s'accompagne "d'une profonde indécision quant à la définition d'une carrière professionnelle $e^{20 "}$.

"Faire carrière", voilà une expression courante qui perd de sa signification quand on n'accède pas à la stabilité de l'emploi et quand on multiplie les expériences de travail sans rapport les unes avec les autres. C'est pourtant une expression qui, durant la jeunesse des enquêtés à Bamako, reflétait l'itinéraire professionnel de leurs parents, à l'image du père de Demba qui "a été avocat toute sa vie”. Pour mes interlocuteurs, la précarité d'emploi entraîne nécessairement une redéfinition du modèle d'intégration professionnelle qui avait été suivi par la génération de leurs ascendants. Ce décalage entre les conditions d'emploi des parents au Mali (position bien intégrée dans le marché du travail) et celles de leurs enfants en France (mar- 
quées par l'intermittence) fait que l'on peut parler d'un déclassement à la fois générationnel et professionnel.

\section{Non-reconnaissance et invalidation juridique des diplômes}

"Tu es médecin, tu es pharmacien, tu es ingénieur, cela veut dire que tu appartiens déjà à un rang, à une classe sociale. Tu ne dois pas être vu dans telle chose, tu ne dois plus faire telle connerie, tu dois être l'image idéale même du quartier. Chez nous [à Bamako], c'est comme ça" (Ibrahim, 38 ans, docteur en pharmacie). Les professions citées par Ibrahim, dites "libérales", sont celles qu'exercent les pères des enquêtés à Bamako. En France, une partie importante de ces métiers est fermée aux étrangers, soumise à la double condition de nationalité et/ou d'être titulaire d'un diplôme d'État français ${ }^{21}$. Ainsi, et pour continuer avec Ibrahim, cet enquêté a tout simplement vu son doctorat de pharmacie malien invalidé en France ${ }^{22}$. Aujourd'hui préparateur en pharmacie, il entre dans la catégorie des professions
Mêlant des logiques sociales,

économiques et affectives,

les envois d'argent au pays

signifient que l'on participe

(enfin) au système

de protection microsocial

fondé sur la parenté. intermédiaires sans pour autant exercer un métier équivalent à sa formation initiale.

L'invalidation juridique de certains diplômes étrangers n'est pas seulement la négation d'une disposition sociale pour les enquêtés concernés. Elle est précisément ce qui les empêche d'assurer la continuité entre les conditions socioéconomiques de la société malienne et celles de la société française. Or, pour parvenir à une aisance économique et sociale à Paris analogue à celle de leurs familles bamakoises, pour "avoir une situa- tion", il leur faudrait avoir accès libre aux classes supérieures françaises. Ce n'est pas le cas. Promotion par le diplôme, faire carrière, avoir une situation, ces trois éléments étaient au centre des stratégies de reproduction sociale des enquêtés avant que ces derniers ne correspondent plus aux opportunités du marché du travail malien. Or, pour la majorité d'entre eux, ces éléments n'ont pas trouvé les conditions de leur mise en œuvre en France. On peut donc parler de déclassement, à condition de se souvenir que la mobilité sociale descendante avait déjà commencé au Mali.

\section{Retour sur la dette intergénérationnelle}

Il reste à aborder un enjeu sociologique majeur de l'immigration des enquêtés : celui de s'acquitter de la dette entre les générations ${ }^{23}$. C'est en enclenchant la mécanique des transferts financiers qu'il devient possible d'honorer les termes du contrat intergénérationnel. Mêlant des logiques sociales, économiques et affectives, les envois d'argent au pays signifient que l'on participe (enfin) au système de protection microsocial fondé sur la parenté. Encore faut-il se rappeler que le "prix de la dette" s'établit selon la condition sociale originelle de mes interlocuteurs, la petite bourgeoisie. Aussi les transferts d'argent vers Bamako visent-ils, pour le mieux, à améliorer la condition de la famille et, pour le moins, à tenir son rang dans l'espace social malien $^{24}$.

D'après mes interlocuteurs, les sommes envoyées à la famille résidant à Bamako varient entre 65600 francs CFA (100 €) et 328000 francs CFA (500 €) par mois. Le montant le plus élevé qui m’ait été annoncé - près de dix fois le SMIG malien découle de l'organisation des cinq frères D. : "Chaque fin de mois, mes frères me donnent $100 €$. Je rajoute 
ma part et j'envoie le tout au pays, au papa." La somme d’argent destinée mensuellement aux parents de la fratrie D. équivaut aux revenus du travail parmi les plus élevés du Mali, une somme qui, sans le moindre doute, maintient la condition sociale de leur famille à Bamako. Ce point est important car il montre que la situation de déclassement vécue par la plupart des enquêtés en France est relative. En effet, les transferts d'argent ascendants (des enfants aux parents), lorsqu'ils existent et qu'ils se conforment aux exigences petites bourgeoises de la dette intergénérationnelle, permettent à bon nombre de mes interlocuteurs de rouvrir les portes de la mobilité familiale et de conquérir leur statut d'adulte. Au déclassement socioprofessionnel en France correspond donc un reclassement familial au Mali.

\section{Conclusion}

Je voudrais avant tout souligner l'intérêt et les difficultés d'une approche empirique de la migration en termes de classes sociales. Du côté de la société française, les enquêtés ont été positionnés à partir de deux critères : la profession et le salaire. Il en est ressorti qu'ils se situaient soit dans la classe populaire, soit dans la classe moyenne inférieure. Cette opération de classement soulève quelques questions, en particulier en ce qui concerne la classe populaire. Pour Olivier Schwartz, la classe populaire présente trois caractéristiques fondamentales : l'insécurité des conditions d'existence, le statut social bas et réputé inférieur, et la distance vis-à-vis des formes de savoirs légitimes et dominants (notamment ceux qui se rapportent au capital scolaire $)^{25}$. C'est ce dernier élément qui pose problème puisque mes interlocuteurs sont précisément porteurs d'un capital scolaire. Si les emplois occupés par les enquêtés sont, pour la plupart, des emplois du bas de l'échelle, peut-on parler, à propos de la position sociale de ces migrants diplômés en France, d'une appartenance aux groupes populaires?

Du côté de la société malienne, parler de classes sociales et de petite bourgeoisie soulève aussi plusieurs interrogations : combien y a-t-il de petits bourgeois au Mali ? Qui inclure ? Qui exclure ? S'agit-il d'“une partie complémentaire de la société bourgeoisie 26 " ? Ces questions sont d'autant plus délicates que les chercheurs semblent s'accorder sur l'aspect inachevé des classes sociales en Afrique subsaharienne ${ }^{27}$. Pourtant, les familles d'origine des enquêtés combinent deux modes de reproduction sociale : le premier est fondé sur la dette intergénérationnelle, le second introduit une composante scolaire qui est précisément "générateur de classes sociales ${ }^{28 ”}$. C'est aussi là que réside le dernier avantage d'une approche de la migration en termes de classes sociales : celui de permettre la comparaison entre la condition sociale d'origine des enquêtés et celle qui est la leur à Paris. Autrement dit, parler de classes sociales, c'est établir un lien entre la société d'émigration et la société d'immigration, c'est se donner une chance supplémentaire de rendre intelligibles les itinéraires des migrants en tenant compte de leurs origines sociales. Pour conclure sur la question du déclassement des immigrants, il faut dire que ce phénomène dépasse largement le cadre des Bamakois diplômés rencontrés à Paris.

À partir d'une enquête statistique et comparative sur l'immigration dans plusieurs pays de l'OCDE (dont la France), Jean-Christophe Dumont et Olivier Monso montrent que les immigrants sont effectivement plus exposés au déclassement professionnel que les autochtones ${ }^{29}$.

Cela ne relève-t-il pas du mode de recrutement et de gestion des migrants sur le marché internationalisé du travail ? 